\title{
Clavicular non-union as an undiagnosed cause of progressive limb-threatening upper extremity ischemia
}

\author{
Łukasz Dzieciuchowicz, Arkadiusz Krzemiński \\ Department of Vascular Surgery and Vascular Diseases, University of Zielona Góra, Poland
}

\begin{abstract}
A rare case of progressive upper limb-threatening ischemia is presented. The ischemia was caused by the chronic embolization from the axillary artery that was compressed by the clavicular pseudoarthrosis due to the remote in time fracture.
\end{abstract}

Key words: clavicular pseudoarthrosis, upper limb ischemia, arterio-arterial embolism

Acta Angiol 2020; 26, 3: 144-146

\section{Introduction}

In comparison to lower extremity, upper limb is less often affected by both acute and chronic limb ischemia. Also, the progression of disease to limb-threatening ischemia is less frequent. The etiology is also different, while the atherosclerosis prevails in lower limb, the upper limb ischemia is most commonly caused by vascular trauma and cardiogenic embolism [I]. That is why the limb-threatening ischemia and its etiology may be misdiagnosed and adequate treatment delayed. The purpose of this report is to present a case of progressive upper limb-threatening ischemia due to chronic intermittent compression of the axillary artery by the clavicular pseudoarthrosis.

\section{Case report}

A sixty-two-year old male was referred to the Department of Vascular Surgery because of severe rest pain and gangrene of stumps of the fingers of the right upper limb. He was a retired truck driver and heavy cigarette smoker. Four months earlier he presented to the general surgery department because of infected necrosis of the fingers of right hand. At that time the infected and necrotic fingers were incised and drained.
Due to lack of improvement and progression of necrosis ischemia was suspected, and the patient was referred to a vascular surgeon. The ischemia was confirmed. Thromboembolectomy of brachial and forearm arteries was performed together with the forearm fasciotomy. Good inflow and outflow were obtained and a remarkable improvement of perfusion of the limb was observed. At that time no atrial fibrillation was observed, and cardiogenic embolism was excluded. The patient was referred back to general surgery department for the amputations of the necrotic phalanges.

At the present hospitalization the limb was critically ischemic and axillary, brachial and distal pulses were absent. A resilient bulge was noted in the supraclavicular area (Fig. I). Thorough history-taking revealed fractures of the right clavicle at three occasions at the age of 12 that is 50 years earlier. Plain X-ray showed the presence of clavicular non-union with its deformation (Fig. 2). Computed Tomography Angiography (CTA) showed patent and free of stenosis brachio-cephalic trunk and subclavian artery, severe stenosis of the proximal portion of axillary artery with patency of its distal segment, brachial artery was stenosed in its proximal segment and occluded distally and the take-off of deep brachial artery was also occluded. The stenosis of the axillary artery 


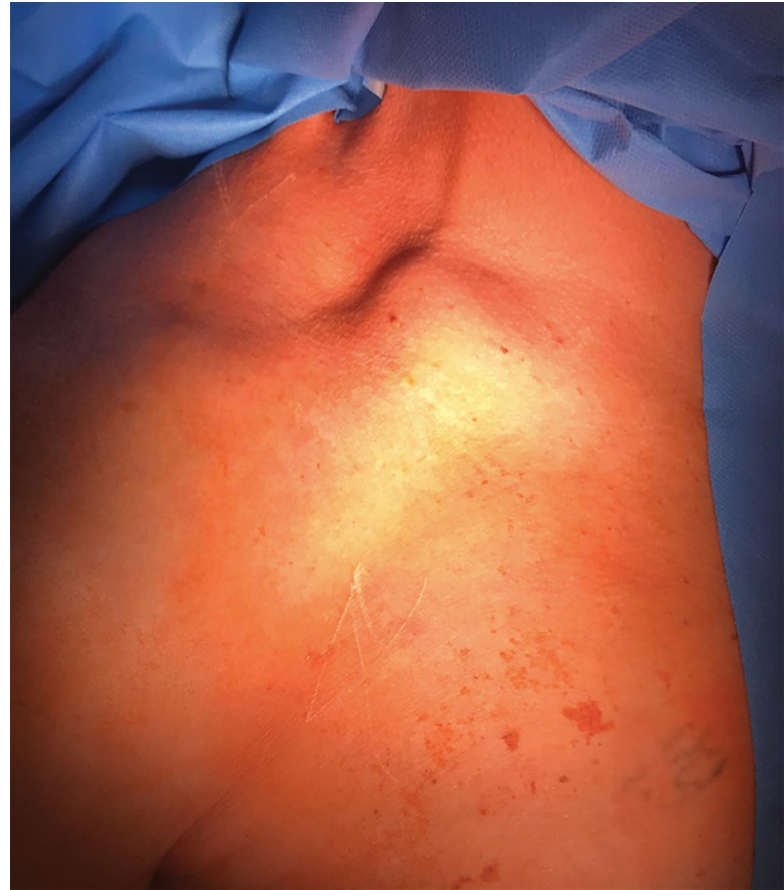

Figure I. A resilient bulge was observed in the supraclavicular area

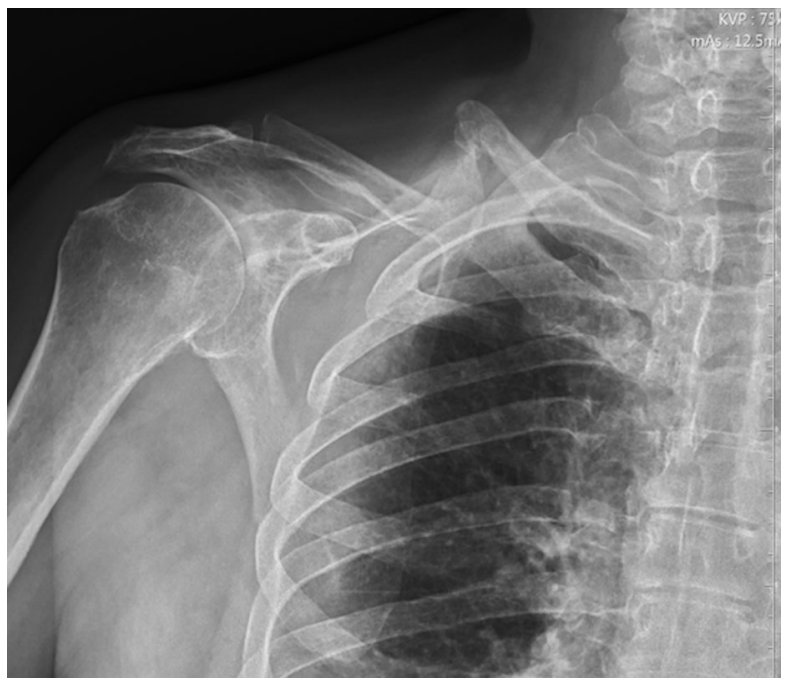

Figure 2. A clavicular non-union with its deformation seen on plain X-ray

was exactly at the site of the clavicular pseudoarthrosis (Fig. 3). The patient was qualified for an urgent surgery. Through an S-shape incision the deformed clavicle together with the pseudoarthrosis was removed. That allowed a good exposure of the axillary artery which was dilated. After longitudinal arteriotomy a thickening of arterial wall and the presence of old thrombi were observed. The lumen was not dilated. With Fogarty

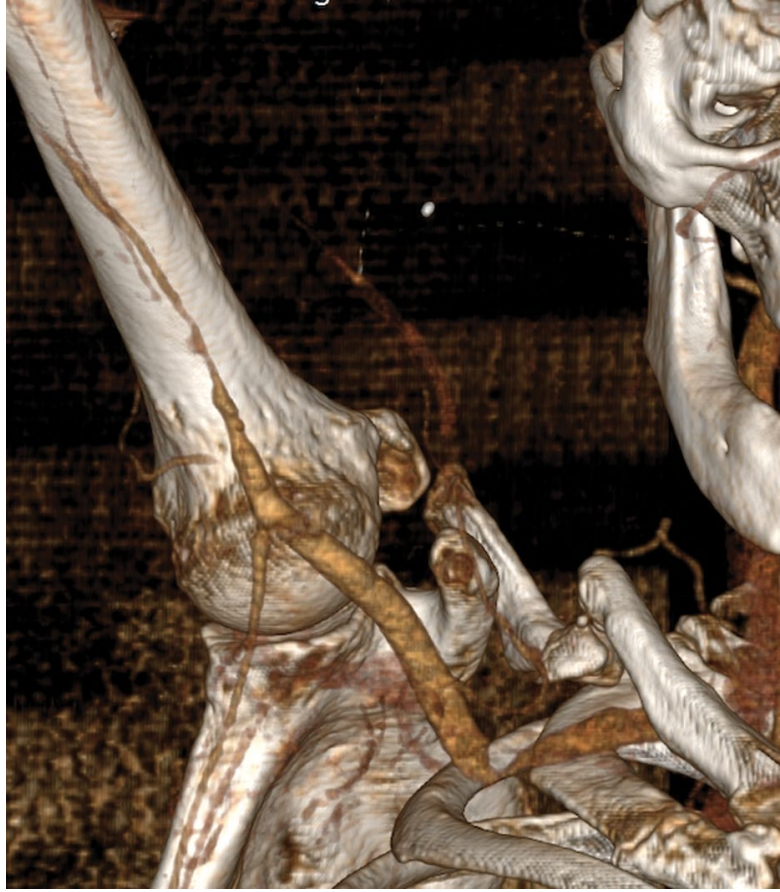

Figure 3. The stenosis of the axillary artery at the site of the clavicular pseudoarthrosis and occlusion of brachial artery seen on the pre-operative CTA

catheter similar old thrombi were removed from the distal axillary artery and proximal brachial artery and a good inflow and outflow were observed (Fig. 5). It was not possible to advance the Fogarty catheter more distally. The arteriotomy was closed with a continuous $5 / 0$ polypropylene suture and the wound was drained and sutured in a layered fashion. Postoperatively, the rest pain subsided and a marked improvement of the perfusion of the limb was observed. A CTA showed widely patent subclavian, axillary and proximal brachial and profunda brachial arteries, the middle portion of brachial artery was occluded and the flow in its distal portion and forearm arteries was reconstituted through collaterals from profunda brachial artery (Fig. 4). The necrotic portions of stumps of the fingers were reamputated and primary healing was achieved. In the six months follow-up no recurrence of limb-threatening ischemia was observed.

\section{Discussion}

This report presents a case of a misdiagnosed upper chronic limb-threatening ischemia. Initially the infected gangrene of the fingers was not associated with limb ischemia and then though the ischemia was diagnosed and treated its cause was not established that led to its recurrence. In the absence of cardiogenic cause of 


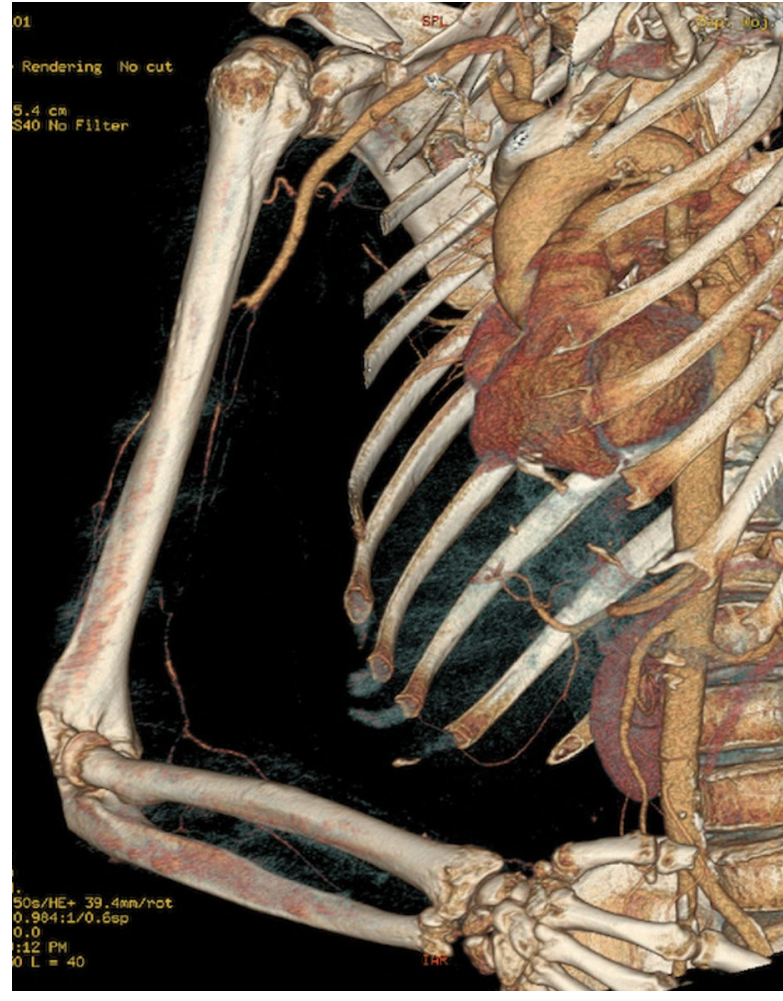

Figure 4. A post-operative angiography

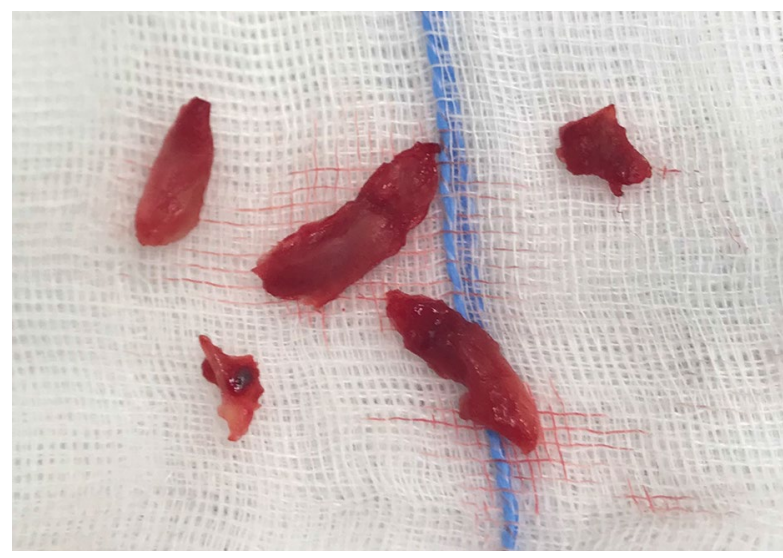

Figure 5. Old thrombotic material removed from the axillary artery and proximal part of brachial artery

emboli, a post embolectomy angiography should have been performed to look for a non-cardiogenic source. The arterial compression by the pseudoarthrosis of the clavicle is a rare but already described cause $[2,3]$. Interestingly, there was a 50-year interval between clavicular fracture and the occurrence of limb threatening ischemia. Similarly, in recent single case reports limb ischemia developed 43 and 30 years after fracture of the clavicle [2, 3]. That long interval may have contributed to a failure to associate the symptoms with the presence of clavicular pseudoarthrosis. In the described case, a chronic repetitive compression of the axillary artery by the clavicular pseudoarthrosis lead to its inflammation, as reflected by the thickening of its wall and thrombosis, which was the cause of progressive embolization of the brachial artery. The chronicity is supported by the presence of very well-developed collateral circulation from the profunda brachial artery as well as by the impossibility of thrombus removal from the middle portion of the brachial artery. It may somehow resemble an arterial thoracic outlet syndrome. However, in the latter case typically a post-stenotic aneurysm is the source of emboli. That was not the case in presented patient that points to an intermittent and not permanent compression of axillary artery.

The thrombectomy of axillary artery and proximal portion of brachial artery removed a source of potential emboli, restored good inflow and re-perfused the deep brachial artery that resulted in a significant improvement in the blood flow to the limb. In case when limb perfusion was unsatisfactory a bypass to the distal brachial artery should be considered.

One of the drawbacks that limit the interpretation of angiographic finding is lack of the preoperative angiography with the upper limb in an anatomical position and of the postoperative angiography with the elevated limb. To prevent the exposure of the patient to the iodinated contrast media and ionizing radiation in a short period of time an additional angiography was not performed. However, we believe that stenosis of axillary artery observed with limb elevation is always pathological.

In conclusions, in case of upper limb ischemia its causes should be thoroughly investigated. Failure to establish the etiology of ischemia will result in recurrence and further deterioration of the condition of the extremity. History of the fracture of the clavicle, even if remote, should draw attention to the possible arterial compression syndrome.

\section{Conflict of interest}

None.

\section{References:}

I. Deguara J, Ali T, Modarai B, et al. Upper limb ischemia: 20 years experience from a single center. Vascular. 2005; 13(2): 84-91, doi: 10.1258/rsmvasc. 13.2.84, indexed in Pubmed: 15996362.

2. Miyamotto M, Sanvido LV, Brendolan LF, et al. Late presentation of critical upper limb ischemia caused by pseudarthrosis of the clavicle. J Vasc Bras. 2018; 17(2): 174-177, doi: 10.1590/16775449.009617, indexed in Pubmed: 30377431.

3. Stella M, Santolini E, Briano S, et al. Late recurrent peripheral upper limb ischemia after non-union of a clavicle fracture. Injury. 2015; 46: S3-S7, doi: 10.1016/s0020-1383(15)30035-8. 